\title{
When the leak is weak - how the first-passage statistics of a biased random walk can approximate the ISI statistics of an adapting neuron
}

\author{
T. Schwalger, D. Miklody, and B. Lindner, ${ }^{\mathrm{a}}$
}

Bernstein Center for Computational Neuroscience and Physics Department of Humboldt University, Berlin, Germany

Received 16 July 2013 / Received in final form 13 August 2013

Published online 28 October 2013

\begin{abstract}
Sequences of first-passage times can describe the interspike intervals (ISI) between subsequent action potentials of sensory neurons. Here, we consider the ISI statistics of a stochastic neuron model, a leaky integrate-and-fire neuron, which is driven by a strong mean input current, white Gaussian current noise, and a spike-frequency adaptation current. In previous studies, it has been shown that without a leak current, i.e. for a so-called perfect integrate-and-fire (PIF) neuron, the ISI density can be well approximated by an inverse Gaussian corresponding to the first-passage-time density of a biased random walk. Furthermore, the serial correlations between ISIs, which are induced by the adaptation current, can be described by a geometric series. By means of stochastic simulations, we inspect whether these results hold true in the presence of a modest leak current. Specifically, we measure mean and variance of the ISI in the full model with leak and use the analytical results for the perfect IF model to relate these cumulants of the ISI to effective values of the mean input and noise intensity of an equivalent perfect IF model. This renormalization procedure yields semi-analytical approximations for the ISI density and the ISI serial correlation coeffcient in the full model with leak. We find that both in the absence and the presence of an adaptation current, the ISI density can be well approximated in this way if the leak current constitutes only a weak modification of the dynamics. Moreover, also the serial correlations of the model with leak are well reproduced by the expressions for a PIF model with renormalized parameters. Our results explain, why expressions derived for the rather special perfect integrate-and-fire model can nevertheless be often well fit to experimental data.
\end{abstract}

\section{Introduction}

The theory of stochastic processes has important applications in physics, chemistry, and biology [1]. One important example is the first-passage-time problem, the question

\footnotetext{
a e-mail: benjamin.lindner@physik.hu-berlin.de
} 
when a stochastic process reaches a certain boundary for the first time. This plays a role in such diverse areas as physical chemistry [2], neurobiology [3], or game theory [4] but is also encountered across scientific fields in noise-induced phenomena like stochastic resonance $[5,6]$. While in most of these applications the mean of the time to reach the boundary is the quantity of interest, for other problems the regularity of spiking, quantified e.g. by the standard deviation of the time, is central, e.g. for the phenomena of coherence resonance [7] or coherent noise-induced transport $[8,9]$. Be it the mean or be it the standard deviation - all of these statistics is captured by the first-passage-time density (FPTD), which gives a complete description of the statistics of the single first-passage time.

However, there is interesting statistics beyond the FPTD: most first-passage times are observed in an ordered sequence. Hence, also the correlations between passage times may be worth an inspection because they characterize other aspects of the stochastic dynamics (see e.g. [10,11]). If we associate the arrival at the boundary with a point in time, we can define a stochastic point process, which will be in the case of non-vanishing correlations between the first-passage times a nonrenewal point process (a renewal process is defined by the absence of any statistical dependence among the intervals between subsequent events [12]). Correlations between intervals can arise, for instance, if the process is driven by an external correlated noise or if the system is subject to adaptation processes on a longer time scale.

A striking example for the application of the first-passage-time problem is the interspike-interval (ISI) histogram of a nerve cell (neuron). Neurons show stereotypical electrical discharges (about $100 \mathrm{mV}$ in amplitude and a few $\mathrm{ms}$ in duration), socalled action potentials - or spikes, by which they encode signals and communicate with other nerve cells. Often, they also display spontaneous firing, i.e. without any sensory stimulus, spikes are generated due to various sources of intrinsic noise or noise-like external driving. How to relate the statistics of this spontaneous firing to a stochastic first-passage process has been worked out in the 1960's by Gerstein and Mandelbrot [3] and others [13-15]: essentially, random excitatory and inhibitory postsynaptic potentials or intrinsic channel fluctuations drive the cell's membrane voltage up and down until it reaches a critical threshold, at which a strong positive feedback mechanism leads to spike generation and the reset of the voltage. In the perfect integrate-and-fire (PIF) model the driving fluctuations are approximated as a mean drift $\mu$ plus temporally uncorrelated Gaussian noise $\xi(t)$ that enters the equation with intensity $D$ according to

$$
\dot{v}=\mu+\sqrt{2 D} \xi(t) .
$$

The application of the fire-and-reset rule (after reaching $v_{T}$, a spike is registered and the voltage is reset to $v=0$ ) renders the model non-linear. However, the probability density for the time $T$ between subsequent passages from the reset point (at $v=0$ ) to the threshold (at $v=v_{T}$ ), i.e. the so-called ISI density can be calculated exactly [3] and is given by an inverse Gaussian probability function:

$$
P_{\mathrm{WN}}(T)=\frac{1}{\sqrt{4 \pi D T^{3}}} \exp \left[-\frac{\left(T-v_{T} / \mu\right)^{2}}{4 D\left(v_{T} / \mu\right)^{2} T}\right] .
$$

This is, of course, equivalent to the FPTD for an overdamped Brownian particle to go from location $x=0$ to $x=v_{T}$ under the influence of a constant field of force $F=\mu$ and subject to a temperature $T$ leading to the diffusion coefficient $D=k_{B} T / \gamma$, a problem that has been solved by Erwin Schrödinger in 1915 [16].

Because the state variable (the voltage $v(t)$ ) is reset and the process starts over again after that, we can imagine the values of the times for subsequent first passages from reset to threshold as a sequence of ordered random numbers. For the simple 
model in Eq. (1) the reset of the state variable erases any memory and the driving noise is uncorrelated. Under theses conditions the sequence of first passage times or equivalently the sequence of interspike intervals consist of independent random numbers, constituting a renewal point process.

The simple model Eq. (1) does not explicitly incorporate any of the many ionic currents that flow through the membrane of a neuron: not the spike-generating sodium and potassium currents (here replaced by a simple fire-and-reset rule) nor the slower inhibitory adaptation currents that down-regulate the firing in many neurons [17] and can lead to correlations among interspike-intervals [18].

Despite the short-comings of Eq. (1), it has been shown by comparison to experimental data $[3,19]$ that the interspike-interval histogram of real cells is often well fit by the inverse Gaussian Eq. (2). Recent theoretical work [20] showed, moreover, that this can be extended to the case of a perfect integrate-and-fire model with a spike-triggered adaptation current, although in this case renormalized parameters in Eq. (2) have to be used.

Because, it would be strange to assume that real neurons are perfect integrators, these results suggest that the inverse Gaussian or, more generally, the statistics of the biased Brownian motion is robust against minor modifications of the dynamics and that such modifications can be accounted for by a simple renormalization of the parameters in Eq. (1). A basic but realistic modification of Eq. (1) is to assume at least a small leak current (this comes about mainly by voltage-independent ion channels), yielding a leaky integrate-and-fire (LIF) model

$$
\dot{v}=-\gamma v+\mu+\sqrt{2 D} \xi(t)
$$

where we still apply the same kind of fire-and-reset rule whenever $v$ reaches $v_{T}$ (for a review and further references on this model, see [21]). For this model the ISI density is known in specific cases $[22,23]$; in general, one can also calculate the Laplace transform of the ISI density [24]. Further, with an LIF model that has a strong leak current $\left(\gamma>\mu / v_{T}\right)$, one can model a truly excitable cell that will fire only due to the dynamic noise $\xi(t)$ or other time-dependent input but will be silent if time-dependent input is absent. The case that we will focus on here is that of a mild leak, or, equivalently, of sufficiently strong input $\mu$, in which the model still shows tonic firing even in the absence of noise. This regime seems to be appropriate to model the firing of many sensory cells with a high firing rate.

In this article we inspect a leaky integrate-and-fire model with white noise and a spike-driven adaptation current and ask, how well the ISI density and ISI correlations can be fit by the theory for a PIF model if we renormalize the parameters of the latter model appropriately. Of central interest will be the question, whether a small amount of leak (i.e. a small $\gamma$ ) is sufficient to change ISI density and correlation patterns qualitatively, or whether these statistics are robust against such changes of the dynamics.

Our paper is organized as follows. First, we introduce the integrate-and-fire model with leak, white noise, and a spike-triggered adaptation current in more detail and define briefly the spike train statistics of interest. In Sect. 3 we explain how to obtain renormalized parameters of the PIF model from simple ISI statistics measured in simulations of the LIF model. In Sect. 4, we apply our renormalization procedure to the simple case of a LIF model without adaptation - here, we demonstrate that indeed the method works rather well and gives reasonable approximations to the ISI density, in particular, if the driving noise is moderately large. In Sect. 5, we then turn to the more involved case of a LIF neuron with spike frequency adaptation and show, that also in this case a small leak current can be well accounted for by our renormalization procedure. We summarize our findings in Sect. 6 and discuss how our 
effective PIF theory can be generalized to more general first-passage-time problems with weakly nonlinear drift functions. As an example we show how the ISI density for an overdamped Brownian motion in a strongly inclined periodic potential compares to such a theory.

\section{Model}

We consider a leaky integrate-and-fire neuron with spike frequency adaptation, described by the following Langevin equations

$$
\begin{gathered}
\dot{v}=\mu-\gamma v-a+\sqrt{2 D} \xi(t) \\
\tau \dot{a}=-a+\Delta \tau \sum \delta\left(t-t_{i}\right) .
\end{gathered}
$$

Here, a spike is generated whenever $v(t)$ reaches $v_{T}$ (in the following we set $v_{T}=1$ ), at the same time the voltage is reset to $v(t) \rightarrow v_{R}=0$ and the adaptation is increased by a constant increment $\Delta$ - this is realized by the feedback term in the second equation that involves the spike train $\sum \delta\left(t-t_{i}\right)$ generated by the model. It is evident that the ISIs are just the first-passage times from reset to threshold and that these passage intervals are in general correlated because of the slow feedback variable $a(t)$.

From a long simulation of the model, we can obtain the ISIs, i.e. the differences between subsequent spike times $T_{i}=t_{i}-t_{i-1}$ as an ordered sequence $T_{0}, T_{1}, T_{2} \ldots$ We can then determine the mean ISI $\langle T\rangle$, its variance $\left\langle\Delta T^{2}\right\rangle$, where $\Delta T=T-\langle T\rangle$, and from these two the ISI's relative standard deviation, known as the coefficient of variation $C_{V}=\sqrt{\left\langle\Delta T^{2}\right\rangle} /\langle T\rangle$. Below, we will also use measures derived from higher moments, such as the skewness and the excess kurtosis

$$
\gamma_{\mathrm{s}}=\frac{\left\langle\Delta T^{3}\right\rangle}{\left\langle\Delta T^{2}\right\rangle^{3 / 2}}, \quad \gamma_{\mathrm{e}}=\frac{\left\langle\Delta T^{4}\right\rangle}{\left\langle\Delta T^{2}\right\rangle^{2}}-3,
$$

respectively. Of course, all the information about these moments is described by the ISI's probability density $P(T)$.

Correlations between ISIs can be described by higher-order probability densities. Here, however, we restrict ourselves to the normalized correlation coefficient between intervals that are lagged by $k$, the so-called serial correlation coefficient (SCC)

$$
\rho_{k}=\frac{\left\langle T_{i} T_{i+k}\right\rangle-\langle T\rangle^{2}}{\left\langle T^{2}\right\rangle-\langle T\rangle^{2}} .
$$

This coefficient gives for each lag $k>0$ a number between -1 and 1 and is the most important indicator of nonrenewal spiking [24,25].

\section{Mapping the model to effective PIF models}

To approximate the ISI statistics of the adapting LIF model, Eq. (4), we will use two different approches: First, we will map the model to an effective PIF model without adaptation, for which the ISI density is known analytically. Second, we will map the model to an effective PIF model with adaptation, for which an expression for the serial correlation coefficient exists. 


\subsection{Interspike interval density}

For the model without leak $(\gamma=0)$ and without adaptation $(\Delta=0)$, one can directly solve the Fokker-Planck equation with absorbing boundary condition $[3,16,24]$ by means of different methods (Laplace transform, method of images), yielding the inverse Gaussian distribution Eq. (2). This distribution leads to the following simple expressions for mean and variance of the ISI

$$
\langle T\rangle=v_{T} / \mu, \quad\left\langle\Delta T^{2}\right\rangle=2 D v_{T} / \mu^{3} .
$$

For finite but weak leak and/or adaptation, we may expect that the ISI density will still be close to an inverse Gaussian. If we know the first two moments of the ISIs, we may choose renormalized effective parameters $\mu_{\text {eff }}$ and $D_{\text {eff }}$ in an effective PIF model

$$
\dot{v}=\mu_{\mathrm{eff}}+\sqrt{2 D_{\mathrm{eff}}} \xi(t)
$$

such that it yields the measured mean and variance of the ISIs. This can be achieved by inverting the above formulas [26]:

$$
\mu_{\text {eff }}=v_{\mathrm{T}} /\langle T\rangle, \quad D_{\text {eff }}=\frac{\left\langle\Delta T^{2}\right\rangle \mu_{\mathrm{eff}}^{3}}{2 v_{\mathrm{T}}} .
$$

Our approximation for the ISI density will be simply the inverse Gaussian density

$$
P_{\mathrm{IG}}(T)=\frac{1}{\sqrt{4 \pi D_{\mathrm{eff}} T^{3}}} \exp \left[-\frac{\left(T-v_{T} / \mu_{\mathrm{eff}}\right)^{2}}{4 D\left(v_{T} / \mu_{\mathrm{eff}}\right)^{2} T}\right]
$$

corresponding to the effective PIF model, Eq. (8).

In order to quantify the deviation of this density from the true ISI density, we will employ different measures. First, we will use the rescaled skewness and rescaled kurtosis (excess) from ref. [20]

$$
\alpha_{\mathrm{s}}=\frac{\gamma_{\mathrm{s}}}{3 C_{\mathrm{V}}}, \quad \alpha_{\mathrm{e}}=\frac{\gamma_{\mathrm{e}}}{15 C_{\mathrm{V}}^{2}},
$$

which both attain the value one, if the density is exactly an inverse Gaussian (irrespective of the values for mean and variance) but will deviate from one for other distributions.

Secondly, we measure the difference between the ISI density $P(T)$ of the full model determined by numerical simulations and the inverse Gaussian density $P_{\mathrm{IG}}(T)$ by the Kullback-Leibler divergence, given by

$$
D_{\mathrm{KL}}=\int_{0}^{\infty} \mathrm{d} T P(T) \log _{2}\left(\frac{P(T)}{P_{\mathrm{IG}}(T)}\right) .
$$

Note that if $P(T)$ is estimated from a finite number of simulated ISIs, this measure is always finite even in a situation in which $P_{\mathrm{IG}}(T)$ is the exact probability density (i.e. $\gamma=0, \Delta=0$ ).

\subsection{Interspike interval correlations}

Regarding the serial correlations, we do not expect that the addition of leak alone $(\gamma>0, \Delta=0)$ will induce serial correlations; in fact, the white-noise-driven LIF 
generates a renewal process. In this case, the SCC Eq. (6) is simply given by $\rho_{k}=0$ for $k>0$.

In the presence of adaptation $(\Delta>0)$, however, the ISIs will be correlated because the adaptation current is not reset upon spiking but depends on the previous spike times and thus on previous ISIs. To find an approximation for the SCC, we will again start with the model without leak $(\gamma=0, \Delta>0)$, i.e. the perfect IF model with spike frequency adaptation. For weak noise, the mean ISI is given by $\langle T\rangle=\left(v_{\mathrm{T}}+\tau \Delta\right) / \mu$ [20], which can be inverted to yield an effective base current

$$
\mu_{\Delta}=\frac{v_{\mathrm{T}}+\tau \Delta}{\langle T\rangle}
$$

Put differently, to calculate the SCC for the general case, we can measure the mean ISI $\langle T\rangle$ and then approximate the model by the effective PIF model

$$
\begin{gathered}
\dot{v}=\mu_{\Delta}-a+\sqrt{2 D} \xi(t) \\
\tau \dot{a}=-a+\Delta \tau \sum \delta\left(t-t_{i}\right) .
\end{gathered}
$$

By construction, this model yields the same mean ISI. For weak noise, the recent study [20] derived a formula for the SCC of this model, which attains the form of a geometric series:

$$
\rho_{k}=-\frac{\alpha(1-\vartheta)\left(1-\alpha^{2} \vartheta\right)}{1+\alpha^{2}-2 \alpha^{2} \vartheta}(\alpha \vartheta)^{k-1}
$$

where

$$
\alpha=\exp \left(-\frac{v_{\mathrm{T}}-\tau \Delta}{\tau \mu_{\Delta}}\right), \quad \vartheta=\frac{\mu_{\Delta}(1-\alpha)-\Delta}{\mu_{\Delta}(1-\alpha)-\alpha \Delta} .
$$

We will test in Sect. 5 whether this approximation yields an adequate description of ISI correlations in the general case of leak and adaptation.

\section{LIF model without adaptation current}

We will first consider the case without adaptation. In Eq. (4), this corresponds to setting $\Delta=0$ leading to the standard LIF model given by the one-dimensional dynamics Eq. (3). Deviations from the case without leak current can be quantified by the dimensionless parameter $\hat{\gamma}=\left(\gamma v_{\mathrm{T}}\right) / \mu$. The deterministic dynamics $(D=0)$ changes abruptly at $\hat{\gamma}=1$ : for $\hat{\gamma}<1$, the model shows sustained periodic spiking; this is the so-called supra-threshold regime. In contrast, for $\hat{\gamma}>1$, the model exhibits a stable equilibrium $v=\mu / \gamma$ below threshold. In this so-called sub-threshold regime the neuron is in a quiescent, non-spiking state in the deterministic case and spiking is only possible with the assistance of noise. In the following, we are mainly interested in the supra-threshold regime. For the fluctuation-driven, sub-threshold spiking regime $(\hat{\gamma}>1)$, we do not expect that a description based on an effective PIF model works because the PIF model exclusively operates in the supra-threshold, tonic spiking regime.

For the purpose of testing the inverse Gaussian theory, we determine mean and variance of the ISI from stochastic simulations, although explicit formulas exist in terms of quadratures. We abstain from using these analytical results in order to be consistent with the procedure in the next section for the adapting neuron model, for 

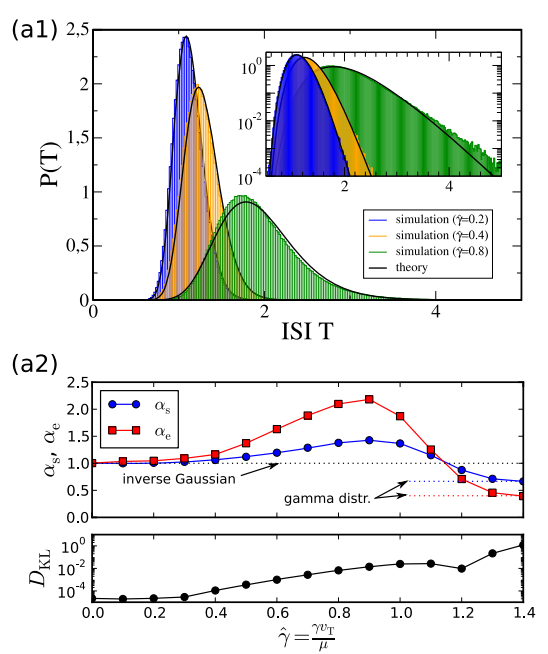

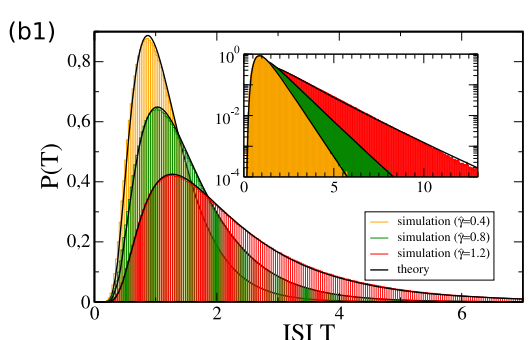

(b2)

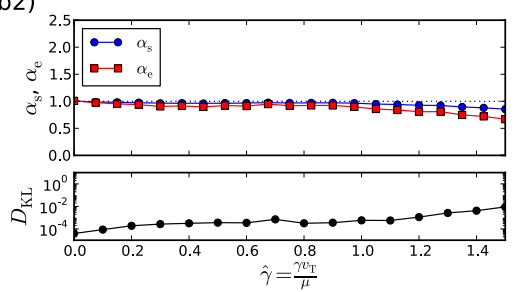

Fig. 1. Interspike interval statistics without adaptation. (a) Weak noise, $D=0.01$ : (a1) ISI densities for different values of $\hat{\gamma}$ as indicated in the legend. Inset: same data in a logarithmic plot. (a2) Rescaled skewness $\alpha_{\mathrm{s}}$, excess kurtosis $\alpha_{\mathrm{e}}$, and Kullback-Leibler divergence $D_{\mathrm{KL}}$ as functions of the non-dimensional leak parameter $\hat{\gamma}=\gamma v_{\mathrm{T}} / \mu$. The black dotted line indicates the case of an inverse Gaussian density. The blue and red dotted lines mark the values $\alpha_{\mathrm{s}}=2 / 3$ and $\alpha_{\mathrm{e}}=2 / 5$ corresponding to a Gamma distribution. (b) Like (a) but tenfold larger noise intensity $(D=0.1)$. The ISI densities (b1) and the rescaled skewness and excess as well as the Kullback-Leibler divergence $D_{\mathrm{KL}}$ (b2) both demonstrate that the deviation from the inverse Gaussian sets in at larger $\hat{\gamma}$. Note that the $\hat{\gamma}$ values in (a1) and (b1) differ.

which explicit analytical results for the ISI moments are not known (see, however, the discussion section, in which we show an example for which analytical results can be used to get a "simulation-free" estimation of the two moments). In any case, the mean and variance of the ISI can be used to calculate the renormalized parameters $\mu_{\text {eff }}$ and $D_{\text {eff }}$ for the PIF theory using Eq. (9). The corresponding inverse Gaussian density, Eq. (10), is compared to model simulations in Fig. 1(a). A visual inspection of the ISI densities yields an excellent fit of the PIF theory in the supra-threshold regime even for the relatively large value $\hat{\gamma}=0.4$.

To compare the ISI statistics of the non-adapting LIF model with the PIF theory more quantitatively, we calculate the rescaled versions of the skewness $\alpha_{\mathrm{s}}$ and excess kurtosis $\alpha_{\mathrm{e}}$ using Eq. (11). For small $\hat{\gamma}$ the values are close to unity, indicating that the LIF model has an inverse Gaussian statistics for sufficiently weak leak current $(\hat{\gamma} \lesssim 0.4$, Fig. 1(a2) for $D=0.01)$. In the supra-threshold regime, the ISI densities becomes slightly more skewed and peaked than an inverse Gaussian as $\hat{\gamma}$ approaches unity. For even larger leak $(\hat{\gamma}>1), \alpha_{\mathrm{s}}$ and $\alpha_{\mathrm{e}}$ decrease and eventually converge to the values $2 / 3$ and $2 / 5$, respectively, corresponding to a gamma distribution. This limit can be understood as follows: in the subthreshold, fluctuation-driven regime spikes are rare events and follow a Poisson statistics [26]. In this case, the ISIs have an exponential probability density, which is a special case of the gamma distribution. Note that gamma-distributed ISIs in the subthreshold regime are in accordance with other studies [27].

Whether the simple renormalization of parameters yields a valid approximation will certainly also depend on the level of noise $D$, which we chose to be small, $D=0.01$ in Fig. 1(a). Remarkably, increasing the noise by a factor of 10 to $D=0.1$ improves the range of validity of the approximation considerably, cf. Fig. 1(b). In this case, the inverse Gaussian yields an excellent fit even in the sub-threshold regime (cf. $\hat{\gamma}=1.2$ 


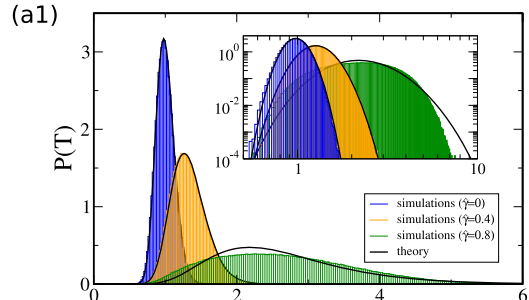

ISI T

(a2)

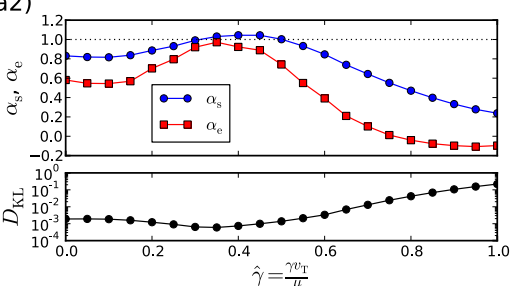

(b1)

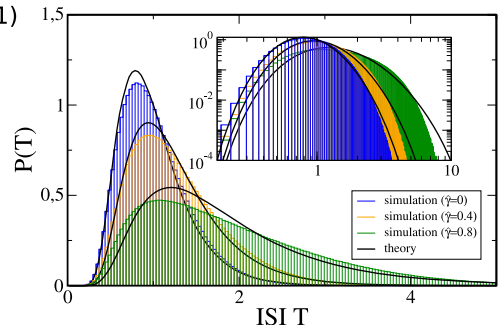

(b2)

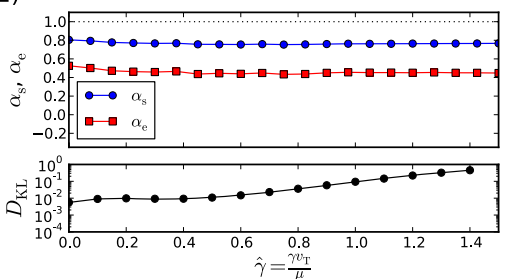

Fig. 2. Interspike interval statistics in the presence of slow adaptation. (a) Weak noise, $D=0.01$ : (a1) ISI densities for different values of $\hat{\gamma}$ as indicated in the legend. Inset: same data in a double-logarithmic plot. (a2) Rescaled skewness $\alpha_{\mathrm{s}}$, excess kurtosis $\alpha_{\mathrm{e}}$, and Kullback-Leibler divergence $D_{\mathrm{KL}}$. The dotted line marks the case of an inverse Gaussian statistics. (b) Corresponding figure for a moderately strong noise intensity, $D=0.1$. Common parameters in all panels: $\mu=4, \tau=10, \Delta=0.3, v_{\mathrm{T}}=1$.

in Fig. 1(b)). A somewhat plausible explanation for this improvement seems to be the linearizing effect of noise, which smears out deterministic bifurcations such as that at $\hat{\gamma}=1$. We should not expect, however, that more noise (e.g. $D=1$ or $D=10$ ) will make the agreement between our approximation and the simulations even better: very strong noise will certainly yield again a stronger disagreement (i.e. $\alpha_{e, s}$ very different from one for already moderately small values of $\hat{\gamma}$ ) because for stronger noise large negative values of the voltage will become more important, values at which the leak current is rather dominant. As a consequence, one may speculate that there is an optimal amount of noise with respect to the range of validity of our approximation.

Our analysis using the skewness and excess is also confirmed by a standard comparison of the probability densities $P(T)$ and $P_{\mathrm{IG}}(T)$, the Kullback-Leibler divergence. As seen in the bottom panels of Figs. 1(a2) and (b2), this measure attains its smallest value at $\gamma=0$ (where the theory is exact) but remains small for a range of positive $\gamma$ values.

\section{LIF model with adaptation current}

Let us now consider the more general case $\Delta>0$, in which the LIF dynamics is influenced by a feedback current mediating adaptation. Figure 2(a1) shows that for slow adaptation and not too large leak $(\gamma \lesssim 0.4)$, the ISI histograms (blue and orange) are close to the inverse Gaussian, Eq. (10). For stronger leak, $\hat{\gamma}=0.8$, the ISI density deviates somewhat. A closer inspection of the skewness and excess kurtosis reveals that for small leak the ISI densities are slightly less skewed and peaked than inverse Gaussian (Fig. 2(a2)). This is not an effect of the leak because already the perfect IF model with adaptation $(\hat{\gamma}=0)$ shows this behavior [20]. At an intermediate range of $\hat{\gamma}$, the ISIs approach the inverse Gaussian statistics when $\alpha_{\mathrm{s}}$ and $\alpha_{\mathrm{e}}$ go through a maximum and the Kullback-Leibler divergence goes through a minimum. For large leak the skewness becomes small and the excess kurtosis becomes negative. This 
(a1)

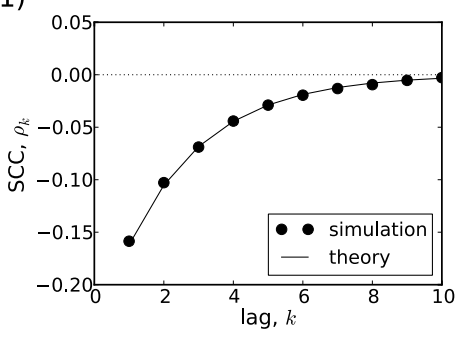

(b1)

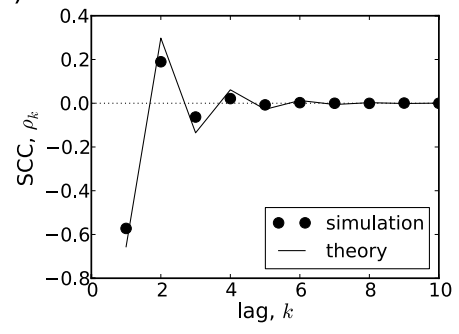

(a2)

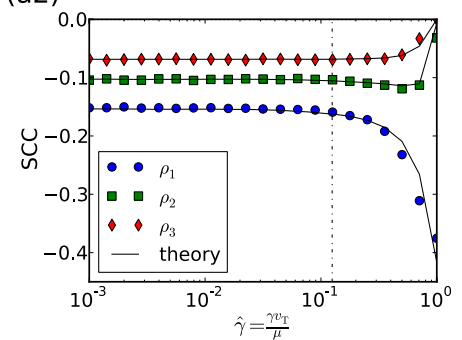

(b2)

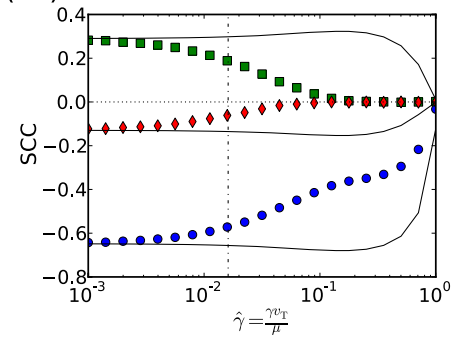

Fig. 3. Serial correlation coefficient (SCC) in the presence of adaptation. (a) Slow and weak adaptation $(\Delta=0.3, \tau=10, \mu=4)$. (a1): The SCC vs. lag for $\gamma=0.5$ shows perfect agreement with the PIF theory, Eq. (15). (a2): The SCC $\rho_{k}, k=1,2,3$, agrees with the PIF theory over a broad range of the non-dimensional parameter $\hat{\gamma}=\frac{\gamma v_{T}}{\mu}$. The location of $\gamma=0.5$ is marked by a vertical dashed-dotted line. (b) Like (a) but for fast and strong adaptation $(\Delta=30, \tau=1, \mu=31)$. (b1) Shows $\rho_{k}$ for $\gamma=0.5$, which is marked by the vertical line in (b2). Parameters: $D=0.01, v_{\mathrm{T}}=1$.

observation corresponds to the shortage of large ISIs as can be seen by the short tail of the ISI density (cf. tails in Fig. 2(a1), inset).

Everything stated above applies to a rather small noise intensity of $D=0.01$. In Fig. 2(b), we show how the statistics changes if we increase the noise intensity tenfold. The agreement between the histograms and the inverse Gaussian is still reasonably good but less impressive than for weaker noise or without adaptation. The interplay of fluctuations and feedback cause a systematic deviation of the rescaled skewness and kurtosis from one (Fig. 2(b2)), a deviation that also becomes apparent in the histograms and in the Kullback-Leibler divergence (bottom panel in (Fig. 2(b2)). Note, however, that noise has again a linearizing effect, as can be seen by the weak dependence of $\alpha_{\mathrm{s}}$ and $\alpha_{\mathrm{e}}$ on the leak parameter $\hat{\gamma}$ (Fig. 2(b2)).

A characteristic effect of adaptation is the emergence of correlations in the sequence of ISIs. For a perfect IF model $(\gamma=0)$ and weak noise, the serial correlation coefficient is approximately given by Eq. (15). Using the renormalized parameter $\mu_{\Delta}$, Eq. (13), with the measured mean ISI of the adapting LIF model, we obtain an approximation of the SCC for the model with leak. Figure 3(a) shows that for slow and moderately strong adaptation, our approximation fits excellently the simulation data in the whole supra-threshold regime $(0 \leq \hat{\gamma}<1)$. In contrast, for fast and strong adaptation, a moderate value of $\hat{\gamma}$ has a strong effect on the magnitude of the SCC, although qualitatively the alternating behavior is conserved (Fig. 3(b)). Note, however, that for moderate $\hat{\gamma}$ the absolute strength of the leak current $\gamma=\hat{\gamma} \mu / v_{\mathrm{T}}$ becomes large because we have chosen a large base current $\mu$ to compensate for the strong adaptation current and thus to maintain a constant firing rate. In absolute terms, a value $\gamma=0.5$ (corresponding to a relatively small value $\hat{\gamma}$ for strong 
adaptation (dashed-dotted vertical line in Fig. 3(b2))) shows a reasonable agreement for both weak and strong adaptation (Figs. 3(a1) and (b1)).

\section{Conclusions}

In this paper we have studied the impact of a finite leak current on the ISI statistics of a tonically firing model neuron. In particular, we found that the firing of a leaky integrate-and-fire model with spike-frequency adaptation can be for weak leak well approximated by a perfect IF model with adaptation if parameters of the latter model are appropriately renormalized. Specifically, the simple renormalization procedure was sufficient to give us a reasonable approximation for the ISI density and ISI correlations for both weak and strong adaptation as long as the leak is weak. Our results explain why the PIF theory can well fit experimental recordings from real neurons $[3,19]$ that are always subject to some finite leak current and possibly other nonlinearities. This indicates that the application of the perfect IF model for modeling spontaneous neural activity is broader than previously thought.

How much leak can we add without obtaining serious deviations of the ISI statistics from the PIF theory? Interestingly, this seems to depend on the intensity of the fluctuations in a nontrivial way. Although, we have not systematically explored the effect but presented only results for two different levels of noise, at least in the absence of adaptation the agreement between theory and simulations was best and hold true for a larger range of the leak parameter $\gamma$ at the higher noise level. At this level, the validity extends - quite unexpected - even into the subthreshold parameter regime. This is due to the linearizing effect of noise. We expect, however, that with a further increase of the noise level the agreement will diminish again at some point. Strong noise will occasionally cause large deviations of the voltage variable towards large negative values, where the leak becomes more important. With adaptation, the situation is more complicated: we observed small deviations from the inverse Gaussian already for the perfect IF model $(\gamma=0)$ in accordance with previous results [20]. For weak noise, these deviations first decrease but then strongly increase upon increasing the leak parameter. For moderately strong noise, the deviations remain small even if we increase the leak considerably, i.e. the not-quite-excellent-but-reasonable approximation to the ISI density can be used for a larger range of $\hat{\gamma}$.

Our results bear relevance for other first-passage-time problems as well and one may generalize our procedure to systems without adaptation but with nonlinear drift functions as follows. Consider the overdamped dynamics of a Brownian particle in a nonlinear potential $U(x)$ with additional constant force $F$

$$
\dot{x}=F-U^{\prime}(x)+\sqrt{2 D} \xi(t),
$$

where the prime denotes the derivative with respect to $x$. In certain situations the statistics of the passage time from $x_{A}$ to $x_{B}$ under the assumption of a weak potential force $-U^{\prime}(x)$ may be of interest. Put differently, our assumption implies that the bias $F$ dominates the passage and that $\left|U^{\prime}(x)\right| \ll F$ for all $x \in\left(x_{A}, x_{B}\right)$.

As an example, we may consider the popular problem of overdamped Brownian motion in a biased periodic potential $[28,29]$ with $U(x)=-a \cos (x)$ in the limit $a \ll F$ and ask for the FPTD to pass one period (e.g. $x_{A}=0$ and $x_{B}=2 \pi$ ). Assuming for simplicity weak noise, we can use standard approximations for the first two moments of the first-passage-time [30] and find in particular for the mean

$$
\langle T\rangle \approx \int_{x_{A}}^{x_{B}} \frac{d x}{F-U^{\prime}(x)}=\int_{0}^{2 \pi} \frac{d x}{F-a \sin (x)}=\frac{2 \pi}{\left[F^{2}-a^{2}\right]^{1 / 2}},
$$




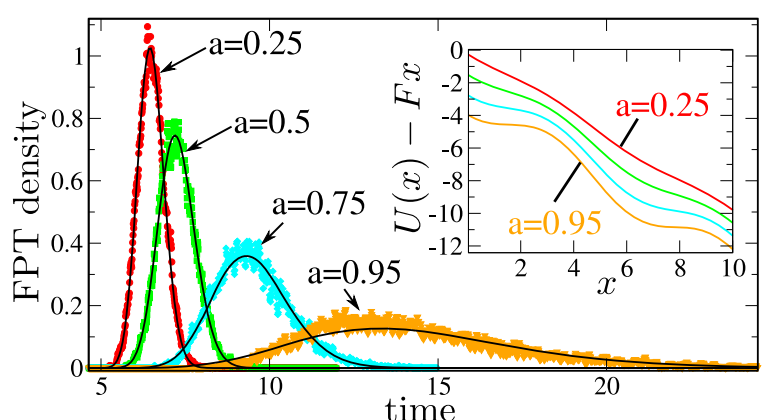

Fig. 4. Comparison of the theoretical expression for the FPT density of a biased random walk model Eq. (2) to simulations of Eq. (17) for a cosine potential $U(x)=-a \cos (x)$, a force of $F=1$ and a noise intensity $D=0.01$; curves are for different values of the potential amplitude as indicated. Parameters of the inverse Gaussian, $\mu_{\mathrm{eff}}$ and $D_{\text {eff }}$ were calculated from Eq. (9) using the approximate first-passage-time formulas Eq. (18) and Eq. (19). Inset: potential shapes of the biased periodic potential for the four different values of the potential amplitude; curves have been shifted vertically for the sake of better visual distinction.

and for the variance

$$
\left\langle\Delta T^{2}\right\rangle \approx 2 D \int_{x_{A}}^{x_{B}} \frac{d x}{\left(F-U^{\prime}(x)\right)^{3}}=2 D \cdot \int_{0}^{2 \pi} \frac{d x}{(F-a \sin (x))^{3}}=\frac{2 \pi D\left(2 F^{2}+a^{2}\right)}{\left[F^{2}-a^{2}\right]^{5 / 2}} .
$$

These expressions can be employed to estimate the effective parameters $\mu_{\text {eff }}$ and $D_{\text {eff }}$ via Eq. (9) and we can then use these parameters in the first-passage-time formula Eq. (2) for the biased random walk. As illustrated in Fig. 4, this approximation then compares very favorably with simulations of the model at low noise intensity $D=0.01$ even for larger values of the ratio of potential amplitude and bias force. This example demonstrates that a moderate nonlinear modulation of the bias can be accounted for by a normalization of parameters of the biased random walk. It would be interesting to explore whether with a nonlinear perturbation $U^{\prime}(x)$ the inclusion of a feedback (similar to the adaptation current in the above neural model) can be additionally incorporated in the approximation for the first-passage-time density.

This paper is dedicated to Vadim Semenovich Anishchenko, on the occasion of his 70th birthday. We acknowledge funding by the BMBF (FKZ: 01GQ1001A).

\section{References}

1. V.S. Anishchenko, V.V. Astakhov, A.B. Neiman, T.E. Vadivasova, L. Schimansky-Geier, Nonlinear Dynamics of Chaotic and Stochastic Systems (Springer, Berlin, 2002)

2. P. Hänggi, P. Talkner, M. Borkovec, Rev. Mod. Phys. 62, 251 (1990)

3. G.L. Gerstein, B. Mandelbrot, Biophys. J. 4, 41 (1964)

4. S. Redner, A Guide to First-Passage Processes (Cambridge University Press, Cambridge, $\mathrm{UK}, 2001$ )

5. L. Gammaitoni, P. Hänggi, P. Jung, F. Marchesoni, Rev. Mod. Phys. 70, 223 (1998)

6. V.S. Anishchenko, A.B. Neiman, F. Moss, L. Schimansky-Geier, Phys-Usp. 42, 7 (1999)

7. A. Pikovsky, J. Kurths, Phys. Rev. Lett. 78, 775 (1997)

8. B. Lindner, M. Kostur, L. Schimansky-Geier, Fluct. Noise Lett. 1, R25 (2001)

9. B. Lindner, L. Schimansky-Geier, Phys. Rev. Lett. 89, 230602 (2002)

10. B. Lindner, Phys. Rev. E 73, 022901 (2006) 
11. B. Lindner, T. Schwalger, Phys. Rev. Lett. 98, 210603 (2007)

12. D.R. Cox, Renewal Theory (Methuen, London, 1962)

13. R.B. Stein, Biophys. J. 5, 173 (1965)

14. R.B. Stein, Biophys. J. 7, 37 (1967)

15. P. Johannesma, Neural Networks, edited by E. Caianiello (Springer, Berlin, 1968), p. 116

16. E. Schrödinger, Physik. Z. 16, 289 (1915)

17. J. Benda, A.V.M. Herz, Neural Comp. 15, 2523 (2003)

18. Y.H. Liu, X.J. Wang, J. Comp. Neurosci. 10, 25 (2001)

19. K. Fisch, T. Schwalger, B. Lindner, A. Herz, J. Benda, J. Neurosci. 32, 17332 (2012)

20. T. Schwalger, K. Fisch, J. Benda, B. Lindner, PLoS Comput. Biol. 6, e1001026 (2010)

21. A.N. Burkitt, Biol. Cybern. 95, 1 (2006)

22. G.E. Uhlenbeck, L.S. Ornstein, Phys. Rev. 36, 823 (1930)

23. A. Bulsara, T.C. Elston, C.R. Doering, S.B. Lowen, K. Lindenberg, Phys. Rev. E 53, 3958 (1996)

24. A.V. Holden, Models of the Stochastic Activity of Neurones (Springer-Verlag, Berlin, 1976)

25. O. Avila-Akerberg, M.J. Chacron, Exp. Brain Res. (2011)

26. R.D. Vilela, B. Lindner, J. Theor. Biol. 257, 90 (2009)

27. S. Ostojic, J. Neurophysiol. 106, 361 (2011)

28. H. Risken, The Fokker-Planck Equation (Springer, Berlin, 1984)

29. P. Reimann, Phys. Rep. 361, 57 (2002)

30. F.T. Arecchi, A. Politi, Phys. Rev. Lett. 45, 1219 (1980) 